\title{
Translesion DNA synthesis by DNA polymerase iota and it's variants
}

\author{
E.S. Shilkin*, A.V. Makarova \\ Institute of Molecular Genetics RAS, Moscow, Russia \\ *shilkinevgeniy.chem@gmail.com
}

Key words: DNA polymerase iota, DNA translesion synthesis, polymorphisms, 8-oxo-G, thymine glycol, AP-site, $\mathrm{O}^{6}$-me-G and 1, $\mathrm{N}^{6}$-ethenoadenine ( $\left.\varepsilon \mathrm{A}\right)$

Motivation and Aim: Genomic DNA is continuously attacked by exogenous and endogenous agents in cells. DNA lesions, if unrepaired, block replication fork progression causing stress. Moreover, DNA damage lead to genomic mutations and related diseases such as cancer. Specialized DNA polymerases are able to bypass lesions in the process called DNA translesion synthesis to overcome replication stress. Human polymerase iota (pol i) is a Y-family DNA-polymerase which as able to bypass a variety of endogenous DNA lesions ( $\left.\mathrm{O}^{6}-\mathrm{me}-\mathrm{G}, \mathrm{TG}, 8-\mathrm{oxo}-\mathrm{G}, \mathrm{AP}\right)$. The structure of pol i active site also stabilizes Hoogsten base pairing between the templating and incoming nucleotides and promotes efficient and accurate DNA synthesis opposite some bulky lesions such as ethenoadenine (eA). Several amino acid polymorphisms of human pol i were recently described (including clinically relevant variants) but their effect on the TLS activity of enzyme is yet to be determined.

Methods and Algorithms: In this work, we analyzed the efficiency and fidelity of DNA synthesis opposite AP-site, thymine glycol, 8-oxo-G, $\mathrm{O}^{6}-\mathrm{me}-\mathrm{G}$ and e-A by several pol i variants: R71G, ?236?, ?251?, ?532?, ?706?. Amino acid substitutions were introduced by site-directed mutagenesis and corresponding proteins were purified from $S$. cerevisiae. Results and Conclusions: We showed that ?236?, ?251?, ?532?, ?706? variants did not significantly affect the TLS activity pf pol i. However, R71G pol i variant dramatically impaired the catalytic activity and changed the specificity of dNTP incorporation opposite DNA lesions. The R71G polymorphism significantly reduced the ability to bypass all tested lesions but eA. In particular, R71G variant incorporated dATP, dTTP and dCTP opposite AP-site and 8-oxo-G with lower efficiency compared to the wild type enzyme. We also observed decrease in incorporation of dCTP opposite $\mathrm{O}^{6}-\mathrm{me}-\mathrm{G}$, TG and eA lesions. Nevertheless R71G variant showed the DNA polymerase activity similar to the wild type enzyme when replicating through eA suggesting that this polymorphic variant supports Hoogsteen interactions during base paring in the active site of pol i. Acknowledgments: This work was supported by RFBR grants No. 17-00-00264 and No. 15-34-70002. 